\title{
Coulomb interaction between a spherical and a deformed nuclei
}

\author{
Noboru Takigawa, * Tamanna Rumin ${ }^{\dagger}$ and Naoki Ihara $\ddagger$ \\ Department of Physics, Tohoku University, Sendai 980-8578, Japan
}

(August 23, 2019)

\begin{abstract}
We present analytic expressions of the Coulomb interaction between a spherical and a deformed nuclei which are valid for all separation distance. We demonstrate their significant deviations from commonly used formulae in the region inside the Coulomb radius, and show that they remove various shortcomings of the conventional formulae.
\end{abstract}

25.70.Jj, 21.60.Ev, 24.10.Eq, 23.20.Js

\section{INTRODUCTION}

The Coulomb interaction between two extended objects is a fundamental quantity in many problems of physics. One example is a heavy-ion collision, where the standard procedure is to approximate the Coulomb interaction between the projectile and target nuclei by simply replacing the target radius by the sum of the projectile and target radii or by the so called Coulomb radius in the formula for the Coulomb interaction between a point charge and an extended target nucleus [1]. This approximation works well as long as the physically relevant region is outside the Coulomb radius. However, one definitely needs to improve the formulae if the region inside the Coulomb radius becomes to play an important role.

In this paper, we present analytic expressions of the Coulomb interaction between a spherical projectile and a deformed target which are valid for any separation distance between them and remove various shortcomings in the standard formulae. Since the formulae would have general values, here we mainly concentrate on derivation of the analytic expressions and comparing with commonly used formulae. The application to actual problems will be reported in separate papers.

In sect. 2 we explain the basic idea of the method. It gives various components of the Coulomb interaction such as the bare Coulomb interaction or inelastic scattering form factors in the linear or higher order couplings in terms of a one-dimensional Fourier integral. In sect.3, we present their analytic expressions obtained by computer assisted performance of the Fourier integrals. In sect.4 we compare the bare potential and the linear as well as the second order coupling form factors calculated by our formulae with those by commonly used formulae by taking the reaction of ${ }^{16} \mathrm{O}$ with ${ }^{238} \mathrm{U}$ as an example. We summarize the paper in sect.5, where we briefly mention some possible systems where the improved formulae will become important.

\section{COULOMB INTERACTION IN THE FOURIER TRANSFORM REPRESENTATION}

Denoting the densities of the projectile and target by $\rho_{P}$ and $\rho_{T}$, the Coulomb interaction between them is given by

$$
V(\vec{R}, \alpha)=\int d \overrightarrow{r_{1}} \int d \overrightarrow{r_{2}} \frac{1}{\left|\vec{R}+\overrightarrow{r_{2}}-\overrightarrow{r_{1}}\right|} \rho_{P}\left(\overrightarrow{r_{2}}\right) \rho_{T}\left(\overrightarrow{r_{1}}\right)
$$

where $\vec{R}$ is the position vector of the center of mass of the projectile measured from that of the target nucleus and describes their relative motion. $\alpha$ represents the ensemble of intrinsic coordinates, which are implicit in $\rho_{P}$ and $\rho_{T}$. They are the deformation parameters in the collective model which we adopt in the following. The key idea is to express the same quantity by the following Fourier transform representation [2, 3],

$$
V(\vec{R}, \alpha)=\frac{1}{2 \pi^{2}} \int_{0}^{\infty} d k \int d \Omega_{k} \int d \overrightarrow{r_{1}} \int d \overrightarrow{r_{2}} \rho_{P}\left(\overrightarrow{r_{2}}\right) \rho_{T}\left(\overrightarrow{r_{1}}\right) e^{i k \cdot\left(\vec{R}+\overrightarrow{r_{2}}-\overrightarrow{r_{1}}\right)}
$$

\footnotetext{
${ }^{*}$ E-mail address: takigawa@nucl.phys.tohoku.ac.jp

${ }^{\dagger}$ E-mail address: rumin@nucl.phys.tohoku.ac.jp

${ }^{\ddagger}$ E-mail address: ihara@nucl.phys.tohoku.ac.jp
} 
For simplicity we assume a uniformly charged object with a sharp surface for both projectile and target, i.e.

$$
\rho_{i}(\vec{r})=\rho_{i}^{(0)} \Theta\left(R_{i}(\Omega)-r\right)
$$

where $\Theta(x)$ is a step function and the index i refers to either the projectile or the target. The angle dependent radius is given in terms of the deformation parameters as

$$
R_{i}(\Omega)=R_{i}^{(0)}\left[1+\sum_{\lambda, \mu} \alpha_{\lambda, \mu} Y_{\lambda, \mu}^{*}(\theta, \phi)\right]
$$

The normalization condition then gives

$$
\rho_{i}^{(0)}=\frac{3 Z_{i} e}{4 \pi R_{i}^{3}} N_{i}(\alpha)
$$

with

$$
N_{i}(\alpha)=\left[1+\frac{3}{\sqrt{4 \pi}} \alpha_{00}^{(i)}+\frac{3}{4 \pi} \sum_{\lambda \mu}\left|\alpha_{\lambda \mu}^{(i)}\right|^{2}+\vartheta\left(\left(\alpha^{(i)}\right)^{3}\right)\right]^{-1}
$$

One usually chooses $\alpha_{00}$ to conserve the volume. In that case, $N_{i}$ is one. In the following, we consider a spherical projectile and a deformed target. Accordingly, we remove the index i to distinguish the projectile or target from the deformation parameters and denote $R_{P}^{(0)}$ and $R_{T}^{(0)}$ simply by $R_{P}$ and $R_{T}$. The integration over $\overrightarrow{r_{2}}$ in eq.(2) can be easily performed, and one obtains

$$
V(\vec{R}, \alpha)=32 \pi \rho_{P}^{(0)} R_{P}^{3} \sum_{\lambda \mu} Y_{\lambda \mu}^{*}\left(\Omega_{R}\right) \int_{0}^{\infty} d k j_{\lambda}(k R) \frac{j_{1}\left(k R_{P}\right)}{k R_{P}} M_{\lambda \mu}^{(T)}(k)
$$

where

$$
\begin{aligned}
M_{\lambda \mu}^{(T)}(k) & =\int d \vec{r} j_{\lambda}(k r) Y_{\lambda \mu}\left(\Omega_{r}\right) \rho_{T}(\vec{r}) \\
& =\frac{\rho_{T}^{(0)}}{k^{3}} \int d \Omega_{r} Y_{\lambda \mu}\left(\Omega_{r}\right) \int_{0}^{k R\left(\Omega_{r}\right)} x^{2} j_{\lambda}(x) d x
\end{aligned}
$$

By expanding with respect to the deformation parameters, one obtains

$$
\begin{aligned}
M_{\lambda \mu}^{(T)}(k)=\frac{\rho_{T}^{(0)}}{k^{3}}[ & \delta_{\lambda 0} \sqrt{4 \pi} \int_{0}^{x} x^{2} j_{0}(x) d x \\
+ & x^{3} j_{\lambda}(x) \alpha_{\lambda \mu} \\
+ & \left.x^{2}\left\{x j_{\lambda}(x)+\frac{x^{2}}{2} \frac{d j_{\lambda}(x)}{d x}\right\} \alpha_{\lambda_{1} \mu_{1}} \alpha_{\lambda_{2} \mu_{2}} \int d \Omega Y_{\lambda_{\mu}} Y_{\lambda_{1} \mu_{1}}^{\star} Y_{\lambda_{2} \mu_{2}}^{\star}+\ldots\right]
\end{aligned}
$$

where $x=k R_{T}$. The first, second and third terms in the square brackets of eq.(10) give the bare Coulomb interaction, the linear and the second order Coulomb couplings, respectively. We thus represent the total Coulomb interaction as

$$
V(\vec{R}, \alpha)=V^{(0)}(R)+V^{(1)}(\vec{R}, \alpha)+V^{(2)}(\vec{R}, \alpha)+\ldots \ldots
$$

where the first three terms are given by

$$
\begin{aligned}
& V^{(0)}(R)=Z_{P} Z_{T} e^{2} F^{(0)}(R) \\
& V^{(1)}(\vec{R}, \alpha)=Z_{P} Z_{T} e^{2} \sum_{\lambda, \mu} F_{\lambda}^{(1)}(R) Y_{\lambda \mu}^{*}\left(\Omega_{R}\right) \alpha_{\lambda \mu} \\
& V^{(2)}(\vec{R}, \alpha)=Z_{P} Z_{T} e^{2} \sum_{\lambda_{1}, \mu_{1}, \lambda_{2}, \mu_{2}} \sum_{\lambda, \mu} F_{\lambda}^{(2)}(R) Y_{\lambda \mu}^{*}\left(\Omega_{R}\right) \alpha_{\lambda_{1}, \mu_{1}} \alpha_{\lambda_{2}, \mu_{2}} \int d \Omega Y_{\lambda \mu} Y_{\lambda_{1} \mu_{1}}^{\star} Y_{\lambda_{2} \mu_{2}}^{\star}
\end{aligned}
$$

with the form factors defined by 


$$
\begin{aligned}
& F^{(0)}(R)=\frac{18}{\pi} \int_{0}^{\infty} j_{0}(k R) \frac{j_{1}\left(k R_{P}\right)}{k R_{P}} \frac{j_{1}\left(k R_{T}\right)}{k R_{T}} d k \\
& F_{\lambda}^{(1)}(R)=\frac{18}{\pi} \int_{0}^{\infty} d k j_{\lambda}(k R) \frac{j_{1}\left(k R_{P}\right)}{k R_{P}} j_{\lambda}\left(k R_{T}\right) \\
& F_{\lambda}^{(2)}(R)=\frac{18}{\pi} \int_{0}^{\infty} j_{\lambda}(k R) \frac{j_{1}\left(k R_{P}\right)}{k R_{P}}\left\{j_{\lambda}\left(k R_{T}\right)+\frac{k R_{T}}{2} \frac{d j_{\lambda}\left(k R_{T}\right)}{d\left(k R_{T}\right)}\right\} d k
\end{aligned}
$$

If we take the rotating frame where the z-axis is chosen to be parallel to the coordinate of the relative motion $\vec{R}$, as is often done in the studies of heavy ion fusion reactions, and if we assume an axially symmetric quadrupole deformation for the target nucleus, then the angular momentum algebra can be explicitly carried out and we obtain the following expressions for the linear and the leading second order Coulomb couplings

$$
\begin{gathered}
V^{(1)}\left(R, \beta_{2}, \theta\right)=Z_{P} Z_{T} e^{2} \sum_{\lambda=2,4,6} F_{\lambda}^{(1)}(R) \beta_{\lambda} Y_{\lambda 0}(\theta, 0) \\
V^{(2)}\left(R, \beta_{2}, \theta\right)=Z_{P} Z_{T} e^{2}\left[F_{\lambda=2}^{(2)}(R) \frac{\sqrt{5}}{7} \frac{1}{\sqrt{\pi}} Y_{20}(\theta, 0)+F_{\lambda=4}^{(2)}(R) \frac{3}{7} \frac{1}{\sqrt{\pi}} Y_{40}(\theta, 0)\right] \beta_{2}^{2}
\end{gathered}
$$

where $\beta_{2}$ is the quadrupole deformation parameter of the target nucleus, and $\theta$ the Euler angle to specify the orientation of its axially symmetric axis in the rotating frame.

The important achievement of the Fourier transform method is that one needs to perform only one dimensional integral, whose results we will present in the next section. Before moving, we wish to comment that one can easily extend the same procedure to incorporate the surface diffuseness of the colliding nuclei by smearing the sharp surface in eq.(33) with a Yukawa function [2.31. The change is simply that the integrand of the Fourier transform representation of each form factor eqs.(15) through (17) gets two additional factors corresponding to the Fourier transforms of the Yukawa function which specify the surface properties of the projectile and target nuclei.

\section{ANALYTIC EXPRESSIONS OF THE BARE COULOMB POTENTIAL AND COUPLING FORM FACTORS}

The Fourier integrals on the r.h.s. of eqs.(15) through (17) are tedious but still doable analytically by hands, since the integrands are given only by polynomials and trigonometric functions. However, computer assisted analytic integration is much easier and practical. Introducing the parameters $R_{C}$ and $R_{C C}$ by $R_{C}=R_{T}+R_{P}$ and $R_{C C}=\left|\left(R_{T}-R_{P}\right)\right|$, the results read,

(1) bare Coulomb interaction,

$$
V^{(0)}(R)=Z_{P} Z_{T} e^{2}\left\{\begin{array}{lrl}
1 / R, & \left(R>R_{C}\right) \\
& {\left[-R^{5}+24\left(R_{P}+R_{T}\right)^{3}\left(-R_{P}^{2}+3 R_{P} R_{T}-R_{T}^{2}\right)+45 R\left(-R_{P}^{2}+R_{T}^{2}\right)^{2}\right.} & \\
\left.+15 R^{3}\left(R_{P}^{2}+R_{T}^{2}\right)-40 R^{2}\left(R_{P}^{3}+R_{T}^{3}\right)\right\} /\left(160 R_{P}^{3} R_{T}^{3}\right) & \\
\left.+\left(R_{P}^{6}-9 R_{P}^{4} R_{T}^{2}+16 R_{P}^{3} R_{T}^{3}-9 R_{P}^{2} R_{T}^{4}+R_{T}^{6}\right) /\left(32 R R_{P}^{3} R_{T}^{3}\right)\right], & \left(R_{C}>R>R_{C C}\right) \\
& & \left(R_{C C}>R>0\right)
\end{array}\right.
$$

These formulae agree with those derived in [4] and used in [5].

(2) quadrupole Coulomb coupling form factor of linear order,

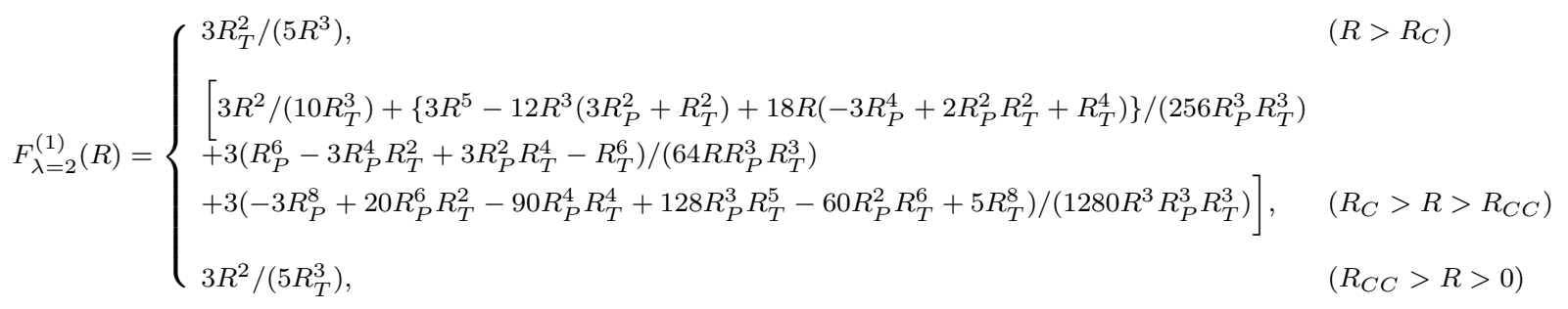


(3) hexadecapole Coulomb coupling form factor of linear order,

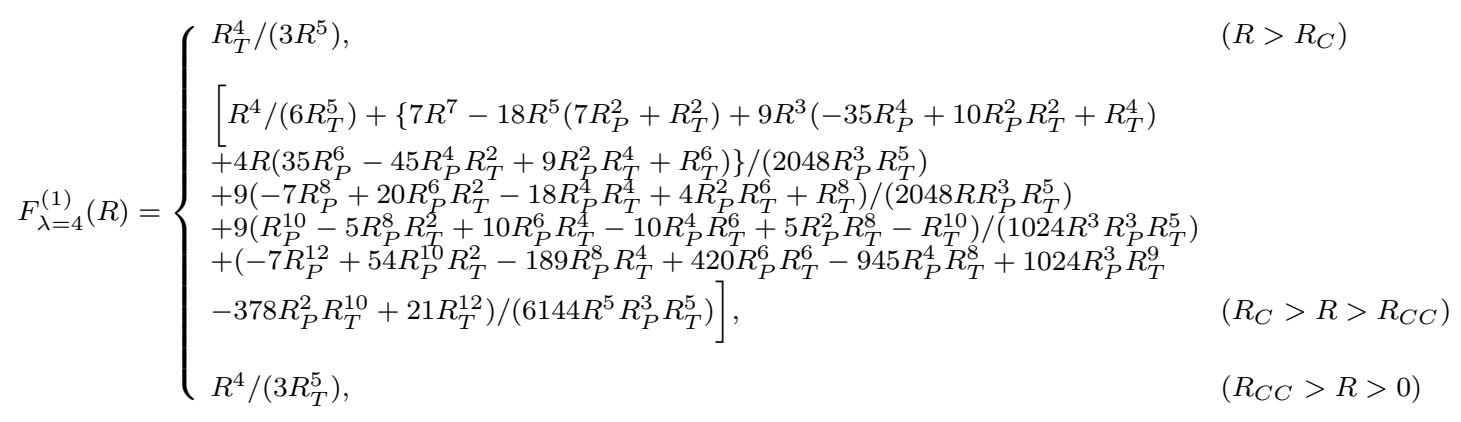

(4) hexacontatetrapole Coulomb coupling form factor of linear order,

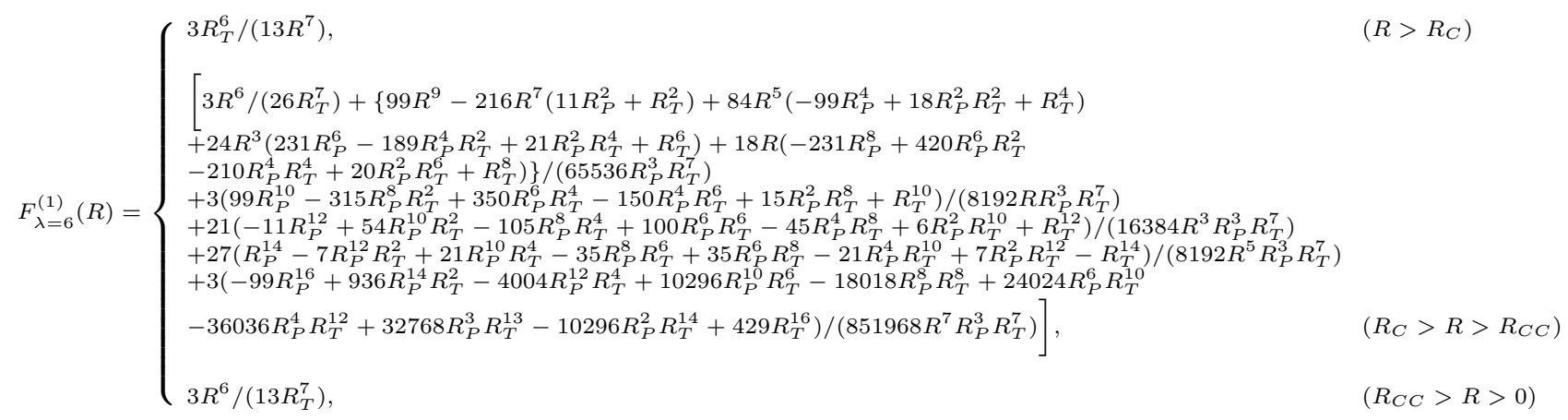

(5) quadrupole Coulomb coupling form factor of second order,

$$
F_{\lambda=2}^{(2)}(R)=\left\{\begin{array}{lr}
6 R_{T}^{2} /\left(5 R^{3}\right), & \left(R>R_{C}\right) \\
{\left[-3 R^{2} /\left(20 R_{T}^{3}\right)-\left\{3 R^{5}-12 R^{3}\left(3 R_{P}^{2}-R_{T}^{2}\right)-18 R\left(3 R_{P}^{4}+2 R_{P}^{2} R_{T}^{2}+3 R_{T}^{4}\right)\right\} /\left(512 R_{P}^{3} R_{T}^{3}\right)\right.} & \\
+3\left(-R_{P}^{6}-3 R_{P}^{4} R_{T}^{2}+9 R_{P}^{2} R_{T}^{4}-5 R_{T}^{6}\right) /\left(128 R R_{P}^{3} R_{T}^{3}\right) & \\
\left.+3\left(3 R_{P}^{8}+20 R_{P}^{6} R_{T}^{2}-270 R_{P}^{4} R_{T}^{4}+512 R_{P}^{3} R_{T}^{5}-300 R_{P}^{2} R_{T}^{6}+35 R_{T}^{8}\right) /\left(2560 R^{3} R_{P}^{3} R_{T}^{3}\right)\right], & \left(R_{C}>R>R_{C C}\right) \\
-3 R^{2} /\left(10 R_{T}^{3}\right), & \left(R_{C C}>R>0\right)
\end{array}\right.
$$

(6) hexadecapole Coulomb coupling form factor of second order,

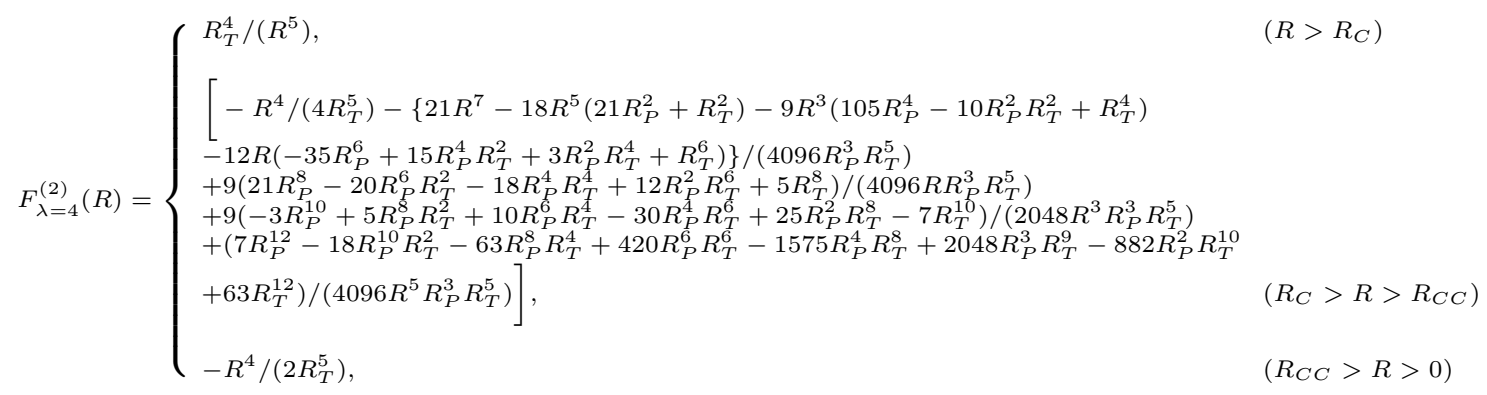

\section{COMPARISON WITH COMMONLY USED FORMULAE}

We now compare our formulae with three commonly used models, which are given by (A) model I (point projectile model) 


$$
\begin{aligned}
V^{(0)}(R) & =\frac{Z_{P} Z_{T} e^{2}}{R} \\
F_{\lambda}^{(1)}(R) & =\frac{3}{2 \lambda+1} \frac{R_{T}^{\lambda}}{R^{\lambda+1}} \\
F_{\lambda}^{(2)}(R) & =\frac{6}{2 \lambda+1} \frac{R_{T}^{\lambda}}{R^{\lambda+1}}
\end{aligned}
$$

(B) model II (uniform charge model 1)

$$
\begin{aligned}
& V^{(0)}(R)=Z_{P} Z_{T} e^{2} \begin{cases}1 / R, & \left(R>R_{C}\right) \\
1 /\left(2 R_{C}\right)\left[3-\left(\frac{R}{R_{C}}\right)^{2}\right], & \left(R<R_{C}\right)\end{cases} \\
& F_{\lambda}^{(1)}(R)=\widetilde{\beta_{\lambda}^{c}} \frac{3}{(2 \lambda+1)} \begin{cases}R_{C}^{\lambda} / R^{\lambda+1}, & \left(R>R_{C}\right) \\
R^{\lambda} /\left(R_{C}\right)^{\lambda+1}, & \left(R<R_{C}\right)\end{cases}
\end{aligned}
$$

with

$$
\widetilde{\beta_{\lambda}^{c}}=\frac{R_{T}^{\lambda} \beta_{\lambda}^{c}}{R_{C}^{\lambda}}
$$

(C) model III (uniform charge model 2)

$$
\begin{aligned}
& V^{(0)}(R)=Z_{P} Z_{T} e^{2} \begin{cases}1 / R, & \left(R>R_{T}\right) \\
1 /\left(2 R_{T}\right)\left[3-\left(\frac{R}{R_{T}}\right)^{2}\right], & \left(R<R_{T}\right)\end{cases} \\
& F_{\lambda}^{(1)}(R)=\frac{3}{(2 \lambda+1)} \begin{cases}R_{T}^{\lambda} / R^{\lambda+1}, & \left(R>R_{T}\right) \\
R^{\lambda} /\left(R_{T}\right)^{\lambda+1}, & \left(R<R_{T}\right)\end{cases}
\end{aligned}
$$

The model I is used in almost all analyses of heavy-ion fusion reactions at energies near and below the Coulomb barrier. The model II can be often found in textbooks of heavy-ion collisions [1]. Model I and II can be too crude concerning the bare potential. The model II and III have a shortcoming that each coupling form factor has a cusp at the separation distance, where the asymptotic formulae are matched to the formulae in the short distance region. The derivative of the coupling form factor is discontinuous at that distance. In writing eqs.(30) and (31), we have assumed that the target nucleus has an axially symmetric static deformation with the Coulomb deformation parameter $\beta_{\lambda}^{c}$. Eq.(31) is the scaling condition of the deformation parameter to guarantee the correct coupling in the asymptotic region.

Figs. 1 through 3 compare the bare Coulomb potential and the linear and quadratic coupling form factors $F_{\lambda=2,4,6}^{(1)}(R)$ and $F_{\lambda=2,4}^{(2)}(R)$ calculated by these formulae and by our improved formulae. The form factors have been multiplied with $Z_{P} Z_{T} e^{2}$ to make the ordinate of all figures have the dimension of energy. They have been calculated for the scattering of ${ }^{16} \mathrm{O}$ with ${ }^{238} \mathrm{U}$. The Coulomb radius parameter has been chosen to be $1.06 \mathrm{fm}$. These figures clearly show the shortcomings of all the three commonly used simple models, which are solved by our new formulae.

The important question is whether the deviations of the conventional models from our improved formulae play some significant roles in actual problems of physics, e.g. in analyzing heavy-ion fusion reactions at energies near and below the Coulomb barrier. In order to have some idea on this question, we again consider the fusion reaction of a spherical projectile ${ }^{16} \mathrm{O}$ with an axially symmetric deformed target ${ }^{238} \mathrm{U}$. It is now well known that the excitation of the ground state rotational band of ${ }^{238} \mathrm{U}$ plays an important role in enhancing the fusion cross section in this reaction. Instead of performing full coupled-channels calculations to take this effect into account, one often describes this reaction based on the no-Coriolis and the sudden tunneling, i.e. degenerate spectrum limit, approximations. In this case, the fusion probability for each partial wave $\mathrm{J}$ is given by first calculating the fusion probability $P_{J}(E, \theta)$ for a fixed orientation of the target nucleus $\theta$ and then taking average over $\theta$

$$
P_{J}(E)=\frac{1}{2} \int_{0}^{\pi} P_{J}(E, \theta) \sin \theta d \theta,
$$

The effective potential for each orientation $\theta$ is given by

$$
V_{J}\left(R, \beta_{2}, \theta\right)=V_{N}\left(R, \beta_{2}, \theta\right)+\frac{\hbar^{2}}{2 \mu R^{2}} J(J+1)+Z_{P} Z_{T} e^{2}\left(F^{(0)}(R)+\beta_{2} F_{\lambda=2}^{(1)}(R) Y_{20}(\theta, 0)\right)
$$


where $V_{N}\left(R, \beta_{2}, \theta\right)$ is the nuclear potential and $\mu$ the reduced mass between the projectile and target. Since our aim is not to perform quantitative analyses, but to illustrate under what circumstances our improved formulae show its power, we took a simple model which considers only quadrupole deformation $\beta_{2}=0.289$ [6] for ${ }^{238} \mathrm{U}$ and linear coupling. The Gauss integral in eq. (34) is replaced by the $\frac{J_{\max }}{2}+1$ points Gauss quadrature if the rotational excitation is truncated at $J_{\max }$

$$
P_{J}(E)=\frac{1}{2} \sum_{i} w_{i} P_{J}\left(E, \theta_{i}\right),
$$

where $\theta_{i}$ are the angles, where the Legendre polynomial $P_{J_{\max }+2}(\theta)$ becomes zero.

The active angles are the zeros of $P_{4}(\cos \theta)$, which are about 30.55 and 70.12 degrees, if $J_{\max }=2$. In Fig. 4 we show the effective s-wave potentials for these two angles calculated by our improved formulae (the solid line) and by three conventional models (the dotted, dashed and dot-dashed lines). We assumed a Wood-Saxon potential for the nuclear potential, where the radius parameter has been chosen such that $R_{N}=R_{N}^{(0)}+R_{T} \beta_{2} Y_{20}(\theta, 0)$ with $R_{N}^{(0)}=R_{P}+R_{T}=1.06\left(A_{P}^{1 / 3}+A_{T}^{1 / 3}\right)$.

We see clear differences among four models. However, these differences do not have any physical significance for ${ }^{16} \mathrm{O}+{ }^{238} \mathrm{U}$ fusion reactions at low energies, since the deviation is localized well inside the barrier region, while the fusion probability is governed by the barrier property, which is the same for all four calculations. Similar situation will hold in general for medium weight heavy-ion collisions. This is a natural consequence of taking the same values for the nuclear and Coulomb radii. We can, however, think of some cases where the difference plays an important role as will be mentioned below.

\section{SUMMARY AND DISCUSSIONS}

We have derived analytic expressions of the Coulomb interaction, which are valid for any separation distance between the projectile and target, and have demonstrated their significant deviations from commonly used models. Our new formulae solve the cusp and discontinuity problems in the form factors and in their derivatives in commonly used models.

We argued that these deviations will not cause any significant effects on the fusion reactions between two heavy nuclei such as the ${ }^{16} \mathrm{O}+{ }^{238} \mathrm{U}$ fusion reactions at energies near and below the Coulomb barrier, which have been very popular subjects of nuclear physics in the past decades. However, we have to keep in mind that this conclusion has been drawn by assuming the same values for the nuclear and Coulomb radii. It is then natural that the deviation of the conventional models from our improved formulae takes place well inside the barrier region. One interesting system will therefore be the system where the Coulomb radius is larger than the nuclear radius. One such example could be heavy-ion collisions induced by unstable neutron deficient isotopes. Another interesting case will be the fusion as well as elastic and inelastic scatterings between light heavy ions, where the absorption in the internal region is not so strong. In this case, the differences among 4 models at short distances will lead to quite different cross sections from each other.

\section{ACKNOWLEDGMENTS}

N.T. wishes to thank Dr. Krappe for useful discussions, and Prof. J. Eichler and his colleagues at the Hahn Meiner Institute, Berlin, for many discussions and kind hospitality.

[1] P. Fröbrich and R. Lipperheide, Theory Of Nuclear Physics, Oxford Science Publication, 1996.

[2] H.J. Krappe, Ann. Phys. 99, 142-163(1976).

[3] Ricardo A. Broglia and Aage Winther, Heavy Ion Reactions, Vol. 1, Elastic and Inelastic Reactions.

[4] Mark W Kermode, Mustafa M Mustafa and Neil Rowely, J. Phys. G: Nucl. Part. Phys. 16, L299-L303(1990).

[5] A.B Balantekin, J.R. Bennett and S. Kuyucak, Phys. Rev. C.49, 1079(1994).

[6] Tamanna Rumin, Kouichi Hagino, Noboru Takigawa, submitted for publication (Physical Review C). 


\section{Figure Captions}

Fig.1

Comparison of bare Coulomb interaction. The solid, dotted, dashed and dot-dashed lines have been calculated based on our improved formulae, Model I through III, respectively.

Fig.2

Comparison of the linear Coulomb coupling form factor plotted in energy scale (see text). Notation is the same as in Fig.1. (a) quadrupole coupling (b) hexadecapole coupling (c) hexacontatetrapole coupling.

\section{Fig.3}

Comparison of the second order Coulomb coupling form factor plotted in energy scale. The solid and the dotted lines are for our improved formulae and Model I.

\section{Fig.4}

Comparison of the effective s-wave barrier calculated based on eq.(35) with our improved formulae and 3 conventional models. The results are shown for two orientations, 30.6 and 70.1 degrees. The lower and the upper barriers correspond to the former and the latter, respectively. 


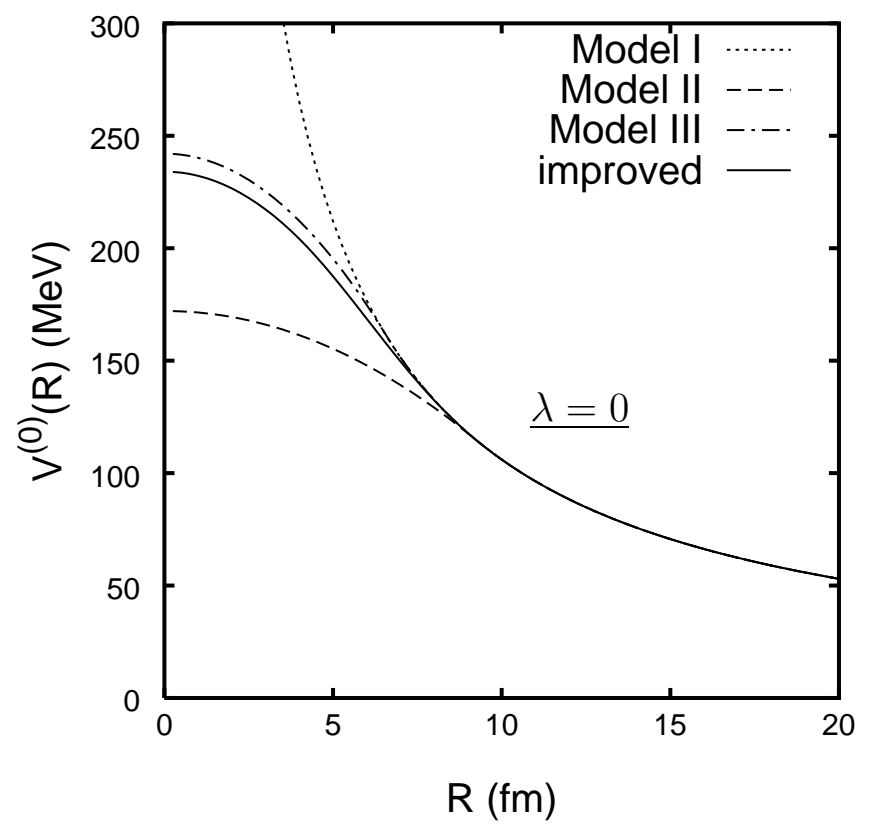

Fig. 1 

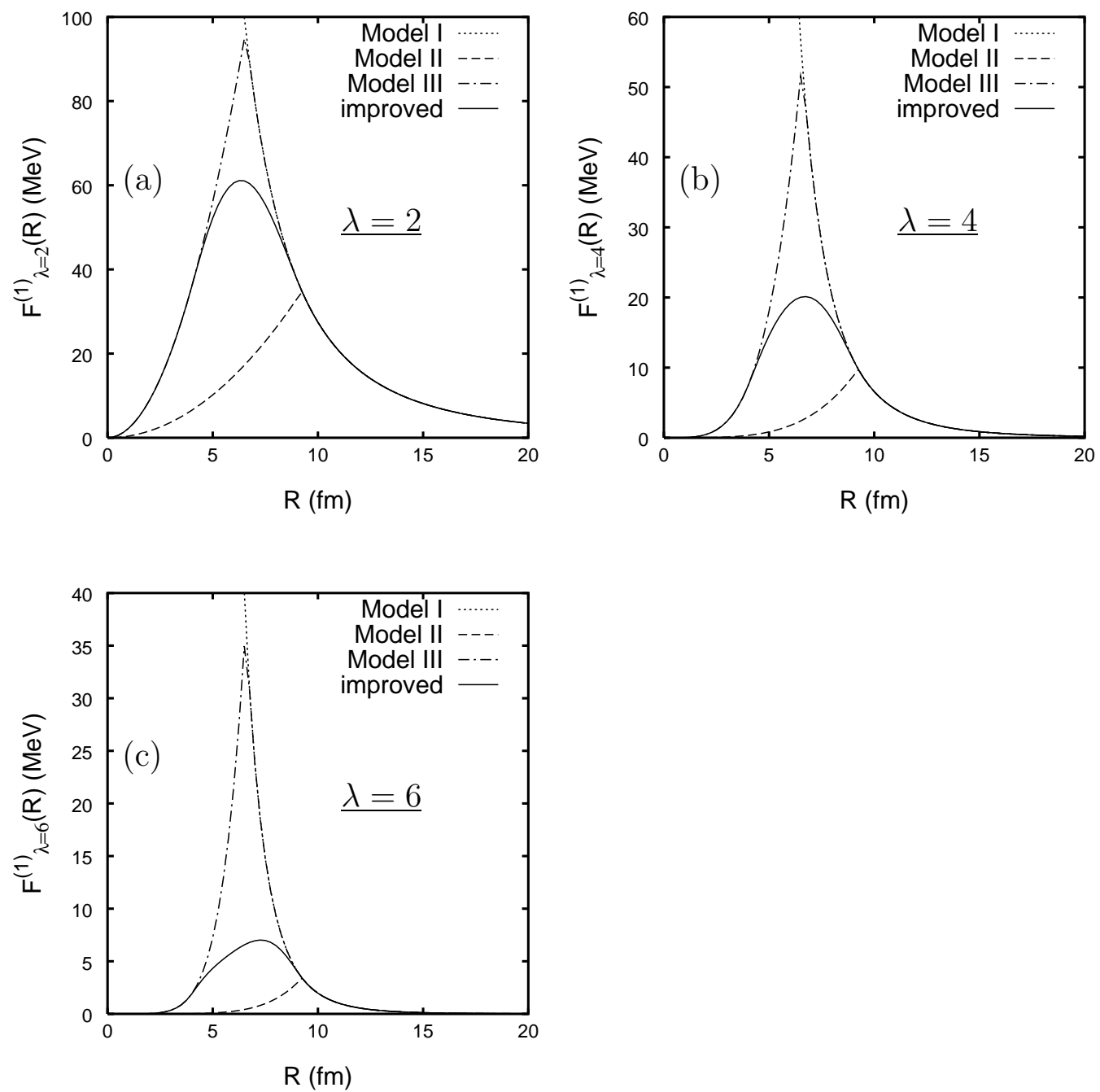

Fig. 2 

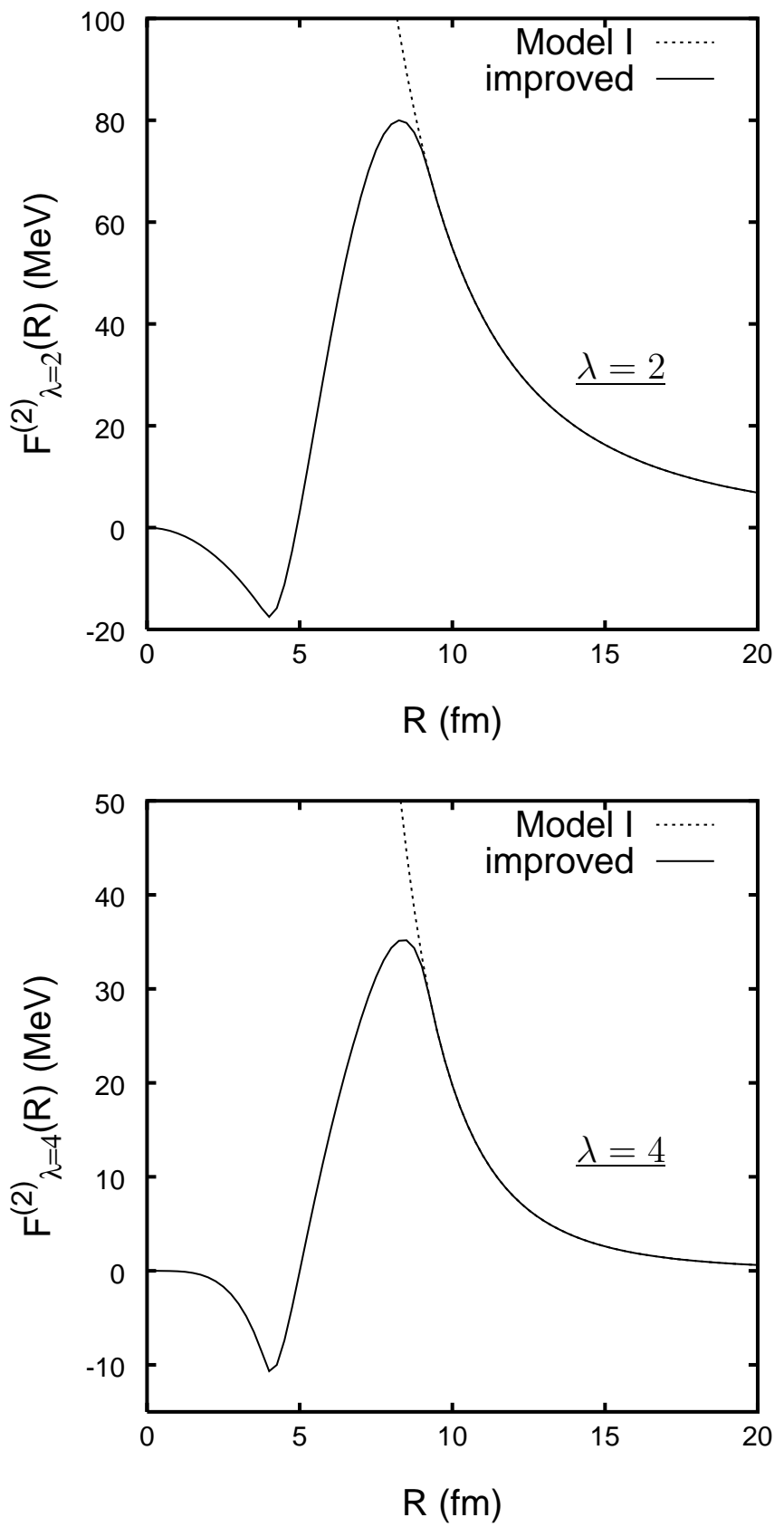

Fig. 3 


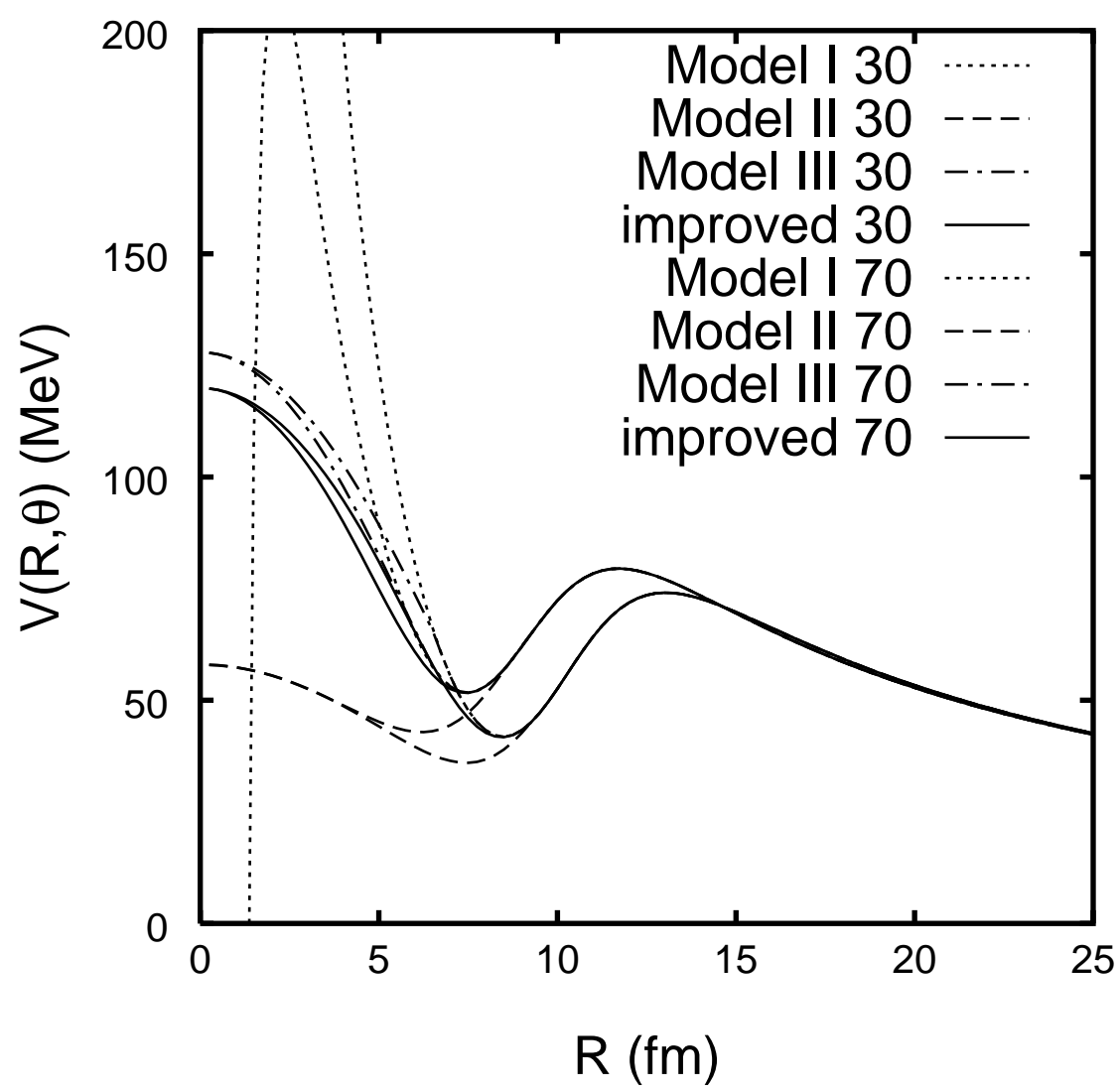

Fig. 4 